\title{
Infectious Mononucleosis Presenting with Loss of Taste and Smell During the SARS-CoV-2 Pandemic?
}

Krishna Nareshkumar Patel, Muhammad Hussain, Amir Khalil, Najeeb Rehman, Hazim Mahdi, Muhammad Jamil Malik, Salim PL Meghjee Barnsley District General Hospital, Barnsley, UK

Received: $10 / 10 / 2020$

Accepted: $28 / 10 / 2020$

Published: $26 / 11 / 2020$

How to cite this article: Patel KN, Hussain M, Khalil A, Rehman N, Mahdi H, Malik MJ, Meghjee SPL. Infectious mononucleosis presenting with the loss of taste and smell during the SARS-CoV-2 pandemic? EJCRIM 2020;7: doi:10.12890/2020_002048.

Conflicts of Interests: The Authors declare that there are no competing interests.

This article is licensed under a Commons Attribution Non-Commercial 4.0 License

\section{ABSTRACT}

A 53-year-old woman presented during the SARS-CoV-2 pandemic with an 18-day history of pyrexia, myalgia, progressive dyspnoea and loss of taste and smell after a close contact had tested positive for SARS-CoV-2. In this period two swabs had been negative for SARS-CoV-2. Clinical examination was normal. During this admission a third SARS-CoV-2 swab was negative, and investigations showed mildly elevated inflammatory markers, mildly deranged liver function, atypical lymphocytes on a blood film and a normal chest x-ray. Her Epstein-Barr virus serology was positive and thus the diagnosis was infectious mononucleosis.

\section{LEARNING POINTS}

- SARS-CoV-2 is not the only virus to cause loss of taste/smell and so other differential diagnoses should be considered.

- Loss of taste/smell is a subjective symptom, and therefore caution should be exercised in the context of an upper respiratory tract infection.

\section{KEYWORDS}

Infectious mononucleosis, taste, smell, SARS-CoV-2

\section{CASE DESCRIPTION}

A 53-year-old woman, with no relevant past medical history, presented to the emergency department with dyspnoea and fever. Her symptoms had originally started 18 days before her presentation, and were accompanied by fatigue, muscle aches and loss of taste and smell. She reported temperatures of up to $40^{\circ}$ using a home thermometer. The symptoms had started a few days after she was in close contact with a friend who had subsequently tested positive for SARS-CoV-2. Soon after her fevers began, she was tested for SARS-CoV-2 with a negative result. When her symptoms persisted for more than 7 days, she presented to the emergency department and was subsequently discharged after another SARS-CoV-2 swab was also negative. She denied any neurological, gastrointestinal or urinary symptoms and did not have any skin changes, weight loss or night sweats. She denied any recent foreign travel. She was not on any regular medications and denied intravenous drug use.

On admission, she was not in respiratory distress, and oxygen saturations and her respiratory rate were normal $\left(\mathrm{SpO}_{2} 98 \%\right.$ on air and 14 breaths per minute, respectively). She was tachycardic on admission with a heart rate of 103 . Her blood pressure was in the normal range $(128 / 72)$ and her temperature on admission was $37.2^{\circ} \mathrm{C}$. Her chest was clear, with normal heart sounds, and there was no lymphadenopathy, no skin changes and no peripheral stigmata of endocarditis, and her abdomen was soft and non-tender. 
Investigations showed a raised lymphocyte count of 5.7×10\%/I (normal range 1.0-3.0×10\%/l), mildly raised CRP (35 mg/l), raised ALT (149 U/l; normal range $0-40 \mathrm{U} / \mathrm{I})$ and AST (137 U/I; normal range 0-40 U/I), and normal GGT and ALP. Ferritin was also raised at 1,168 $\mu \mathrm{g} / \mathrm{I}$ (normal range 22-322). The chest x-ray was reported as clear (no changes compared with the previous study on her presentation to the emergency department 7 days after her symptoms had started). The SARS-CoV-2 swab on this admission was also negative, as were SARS-CoV-2 IgG antibodies. D-dimer was significantly raised at $3.1 \mu \mathrm{g} / \mathrm{ml}$ (normal range $0.05-0.50$ ) and a subsequent CT pulmonary angiogram did not reveal any pulmonary emboli, nor any changes in the lungs.

Further investigations during her admission showed a normal thyroid function and blood-borne virus screen. The blood film showed atypical lymphocytes and her Epstein-Barr virus serology was positive, confirming the diagnosis of infectious mononucleosis.

\section{DISCUSSION}

Infectious mononucleosis is characterized by pharyngitis, cervical lymphadenopathy, fatigue and fever. Given the non-specific nature of symptoms, it cannot be diagnosed by symptoms alone. The hallmark of Epstein-Barr virus, which causes infectious mononucleosis, is the atypical lymphocytes seen on a blood film ${ }^{[1]}$. Similarly, mild symptoms of SARS-CoV-2 also include fever, malaise and sore throat ${ }^{[2]}$. A systematic review found that approximately $60 \%$ of patients with confirmed SARS-CoV- 2 have loss of taste and smell ${ }^{[3]}$.

The symptoms of this patient were highly suggestive of SARS-CoV-2. Given that the sensitivity of SARS-CoV-2 PCR testing is approximately $70 \%{ }^{[4]}$, one negative swab result could be ignored if the pre-test probability is high. However with three negative swabs, the likelihood of the patient having SARS-CoV-2 is extremely small and therefore it is important to explore other differential diagnoses. Other features of the case that suggested an alternative diagnosis to SARS-CoV-2 were a normal chest $\mathrm{x}$-ray and lymphocytosis, as lymphopenia is associated with more severe cases of SARS-CoV-2 ${ }^{[2]}$.

Naturally during the SARS-CoV-2 pandemic, as clinicians we are rightly focussed on detecting cases of COVID-19 in patients who present with viral symptoms. If they did not have access to testing at the time of onset, it is plausible that the symptoms could have been labelled as a 'clinical diagnosis' of SARS-CoV-2. However, it is important to remember that other viruses can also cause loss of taste and smell such as rhinovirus, parainfluenza virus and less commonly, Epstein-Barr virus. While these have all been implicated in causing post-viral olfactory dysfunction in a small number of cases ${ }^{[5]}$, there is no strong evidence to suggest that Epstein-Barr virus also causes loss of taste.

This then raises the subjectivity of the loss of taste/smell described by the patient. Given that pharyngitis is a key feature of infectious mononucleosis, it is entirely plausible that this could have contributed to a sensation of altered, or loss of, taste. Similarly, pure anosmia itself could cause an altered perception of taste sensation, and could therefore be described as a 'loss of taste'.

This case highlights the need for an open mind when dealing with a patient presenting with upper respiratory tract symptoms. Interrogating subjective symptoms such as loss of taste and smell may help widen or narrow the list of differential diagnoses when assessing patients over the coming winter months.

\section{REFERENCES}

1. Balfour HH Jr, Dunmire SK, Hogquist KA. Infectious mononucleosis. Clin Transl Immunol 2015;4(2):e33.

2. Cascella M, Rajnik M, Cuomo A, Dulebohn SC, Di Napoli R. Features, evaluation, and treatment of coronavirus (COVID-19) [Updated 2020 Aug 10]. In: StatPearls [Internet]. Treasure Island (FL): StatPearls Publishing; 2020 Jan-. Available from: https://www.ncbi.nlm.nih.gov/books/NBK554776/

3. da Costa K, Carnaúba A, Rocha KW, de Andrade K, Ferreira S, Menezes PL. Olfactory and taste disorders in SARS-CoV-2: a systematic review. Brazil J Otorhinolaryngol 2020 Jun 9;S1808-8694(20)30066-5. doi: 10.1016/j.bjorl.2020.05.008 [Epub ahead of print].

4. Arevalo-Rodriguez I, Buitrago-Garcia D, Simancas-Racines D, Zambrano-Achig P, del Campo R, Ciapponi A, et al. False-negative results of initial RT-PCR assays for SARS-CoV-2 a systematic review. medRxiv 2020.04.16.20066787. doi: https://doi.org/10.1101/2020.04.16.20066787

5. Suzuki M, Saito K, Min WP, Vladau C, Toida K, Itoh H, Murakami S. Identification of viruses in patients with postviral olfactory dysfunction. Laryngoscope 2007;117(2):272-277. 